\section{Imaging of Neurodegenerative Disorders}

\author{
S.G. Kanekar, ed.
}

New York, NY: Thieme, 2016, 397 pages, $\$ 189.99$

Neurodegenerative diseases comprise a broad swath of different neurologic diseases characterized by loss of neurons in the central nervous system. The most common neurodegenerative disease is Alzheimer disease, a dementing disease. Dementia is commonly understood as loss of function in at least two cognitive domains that is severe enough to affect daily activities in the social or occupational spheres.

Elderly adults represent a significant and rapidly expanding proportion of the population. Some estimates state that by 2030 there will be 72 million individuals over the age of 65 years in the United States, constituting $19 \%$ of the population. The World Health Organization estimated in 2010 that there were 36.5 million people worldwide living with dementia, with the global cost for care being $\$ 604$ billion per year. A new case of dementia is diagnosed approximately every 4 seconds. As the prevalence of many neurologic diseases increases with age, it can be difficult to differentiate between the effects of aging and those of prodromal age-related disease.

Advances in neuroimaging have afforded significant insight into progressive neurodegenerative disorders and their mimics. Besides the improvements in structural imaging that have come about through thinner slices, 3-dimensional volumes, and higher spatial resolution, molecular and cellular imaging have made a big impact on how we look at the brain and its function. MR spectroscopy, diffusion tensor imaging, perfusion imaging, functional MRI, and PET scans have further increased our understanding of the pathophysiology of neurodegenerative disorders. Distinguishing between preventable diseases, potentially reversible diseases, and progressive, irreversible diseases is important in planning for a patient's future medical, social, and economic spheres.
Imaging of Neurodegenerative Disorders is a comprehensive, updated reference covering the latest techniques used in neuroimaging as well as the basic structural imaging that facilitates diagnosis of various neurodegenerative disorders. Because there never has been a dedicated textbook on the imaging of neurodegenerative diseases, the aim of this book is to cover each disease as it is currently understood and to show what it might look like using various imaging techniques. Imaging helps enormously in affirming suspicions, differentiating entities that have a clinical overlap, and charting the progression of neurodegenerative diseases.

The book is organized into 16 parts with 41 chapters written by 83 contributors who have brought fresh insights and expertise that encompass many disease entities. They attempt to fill the gap of knowledge that exists in the imaging and understanding of neurodegenerative diseases. Chapters are arranged by specific diseases and cover their clinical features, pathologic characteristics, and imaging. Along with a brief discussion of each disease are the images themselves - superb in quality and quite illustrative-in addition to what tests to order, what to look for, what one expects to see for each disorder, and what new clinical and research modalities are available.

This book is useful for radiologists, neuroradiologists, neurologists, neurosurgeons, and other internal or family medical physicians, as well as anyone seeking to understand neurodegenerative disorders through the lens of imaging techniques that are becoming increasingly sophisticated.

\author{
E. Edmund Kim \\ University of California at Irvine \\ 101 The City Dr. $S$. \\ Orange, $C A 92868$ \\ E-mail: edmundek@uci.edu
}

Published online Jun. 15, 2016.

DOI: 10.2967/jnumed.116.179218 